
\title{
25 Research Sourere \\ In-Hospital Rehabilitation and Functional Status of Non-Critically ill COVID- 19 Survivors
}

\section{Benjamin Musheyev}

Renaissance School of Medicine at Stony Brook University

Rebeca Janowicz

Renaissance School of Medicine at Stony Brook University

\section{Lara Borg}

Renaissance School of Medicine at Stony Brook University

Michael Matarlo

Renaissance School of Medicine at Stony Brook University

Hayle Boyle

Renaissance School of Medicine at Stony Brook University

Wei Hou

Renaissance School of Medicine at Stony Brook University

Tim Q Duong ( $\square$ Tim.duong@einsteinmed.org )

Montefiore Medical Center and Albert Einstein College of Medicine

\section{Research Article}

Keywords: Coronavirus disease 2019, SARS-CoV2, functional outcome, COVID-19 sequela, long hauler, ICU Mobility, Barthel Index

Posted Date: April 27th, 2021

DOI: https://doi.org/10.21203/rs.3.rs-433175/v1

License: (c) (1) This work is licensed under a Creative Commons Attribution 4.0 International License. Read Full License 


\section{Abstract}

This study investigated in-hospital rehabilitation and functional status at discharge of non-critically ill COVID-19 survivors with respect to pre-admission dependency status, discharge durable medical equipment, discharge medical follow-up recommendation, hospitalization duration, demographics, comorbidities, laboratory tests, and vital signs at hospital discharge. Comparisons were made between COVID-19 survivors who received rehab $(\mathrm{N}=155)$ and those who did not $(\mathrm{N}=162)$. Functional scores were obtained using the "Mental Status", ICU Mobility, and modified Barthel Index scores at hospital discharge. Relative to the non-rehab patients, rehab patients were older, had more comorbidities, had worse preadmission dependency status $(p<0.05)$, were discharged with more assistive equipment and supplemental oxygen $(p<0.001)$, spent more days in the hospital $(p<0.001)$, had more follow-up referrals $(p<0.05)$ with cardiology, vascular medicine, urology, and endocrinology being the top referrals, and had more secondary in-hospital diagnosis of AKI and acute respiratory failure. Functional scores of noncritically ill COVID-19 survivors were impaired at discharge and were associated with pre-admission dependency. Some functional scores were negatively correlated with age, hypertension, coronary artery disease, chronic kidney disease, psychiatric disease, anemia, and neurological disorders $(p<0.05)$. These findings warrant follow up of COVID-19 survivors as many survivors will likely have significant post-acute COVID-19 sequela.

\section{Introduction}

Coronavirus disease 2019 (COVID-19) $(1,2)$ caused by the novel severe acute respiratory syndrome coronavirus 2 (SARS-CoV-2) has evolved into a global pandemic. To date, SARS-CoV2 has killed 3 million and infected 136 million worldwide (https://coronavirus.jhu.edu, Apr 12, 2021). Many SAR-CoV2 infected patients are hospitalized with debilitating illness and some will likely require medium to long term medical treatments and rehabilitation. In-hospital rehabilitation that includes physical and occupational therapy has been shown to minimize hospital-acquired weakness, promote rapid functional recovery, and improve quality of life (3-5). COVID-19 circumstances, however, have made in-hospital rehabilitation challenging for COVID-19 patients, limiting access to the types of restorative services and extent of rehabilitation. During rehabilitation, functional status can be assessed to determine where patients should be discharged, the types of assistive equipment needed, as well as to identify which patients may need further rehabilitative interventions. Common inpatient assessment tools of functional status include Mental status, ICU Mobility Scale, and Barthel Index. Mental status assesses whether a patient is alert and oriented to person, place, time, and situation (6). The ICU Mobility Scale assesses mobility ranging from being passively rolled in bed, to ambulating independently (7). The Barthel Index evaluates the level of assistance required to complete basic activities of daily living (ADL) including feeding, toilet transfers and toileting, bathing, dressing, grooming, and stair negotiation (8). Systematic documentation of functional status of hospitalized COVID-19 survivors at hospital discharge could help to identify patients who may need additional medical or rehabilitative interventions as well as to anticipate future healthcare needs (8-17). To date, data on the extent of in-hospital rehabilitation, functional status, and other 
clinically relevant information at hospital discharge (such as the types of assistive equipment needed, follow-up medical recommendations, and discharge locations) of COVID-19 survivors are generally lacking.

The goal of this study was to investigate the extent of in-hospital rehabilitation and degree of functional status of COVID-19 non-critically ill survivors with respect to pre-admission dependency status, clinical and laboratory test data, length of hospitalization, medical follow-up recommendation, discharged durable medical equipment (DME), discharge location, amongst others. Patients were grouped by those who received in-hospital rehabilitation and those who did not. Mental status, ICU Mobility Scale, and Barthel Index scores were tabulated and correlated with clinical and laboratory variables. To our knowledge, this is the first study to systematically evaluate the rehabilitation and functional status of non-critically ill COVID-19 survivors at hospital discharge and correlate functional status with other clinical variables.

\section{Methods}

This retrospective study was approved by the Institutional Review Board with an exemption for informed consent. This study followed the Strengthening of Reporting of Observational Studies in Epidemiology (STROBE) reporting guidelines for cross-sectional studies (http://www.equator-network.org/reportingguidelines/strobe/). Data were obtained from the emergency room at Stony Brook University Hospital between March 27, 2020 and August 11, 2020. COVID-19 was confirmed based on a real-time polymerase chain reaction test for severe acute respiratory syndrome coronavirus 2 (SARS-CoV-2) on a nasopharyngeal swab specimen. Exclusions were COVID-19 positive patients who were: i) not hospitalized, ii) had incidental COVID-19 findings but were admitted for other major medical indications (i.e., trauma), iii) still in the hospital at the time of the study, iv) less than 18 years old, and v) admitted to the ICU. Electronic medical record data were extracted for 155 patients in the rehab group and 162 patients in the non-rehab group.

Demographics, comorbidities and laboratory variables: Demographics (age, gender, ethnicity, and race), comorbidities, pre-admission dependency status, medical insurance status, laboratories tests and vital signs were tabulated for the rehab and non-rehab group. Chronic comorbidities included smoking, hypertension, diabetes, asthma, chronic obstructive pulmonary disease, coronary artery disease, heart failure, cancer, and chronic kidney disease, amongst others. Symptoms included fever, shortness of breath, cough, myalgia, nausea/vomiting, fatigue, diarrhea, chest discomfort/pain, headache, amongst others. Laboratory tests at hospital admission included C-reactive protein, D-dimer, ferritin, lactate dehydrogenase, lymphocytes, procalcitonin, alanine aminotransferase, aspartate aminotransferase, and troponin, amongst others. Vital signs included heart rate, respiratory rate, pulse oxygen saturation [ $\left.\mathrm{SpO}_{2}\right]$, systolic blood pressure and temperature at hospital admission. In addition, the prevalence of in-hospital acquired acute kidney injury (AKI), acute respiratory failure, and acute respiratory distress were also tabulated. 
Pre-admission dependency: Pre-admission dependency status - extracted from comprehensive care management notes in the medical record - was tabulated as independent, partial assistance or dependent status for the rehab and non-rehab group. Independent patients were those who did not require any physical assistance for mobility or to perform activities of daily living (ADL). Patients in the partial assistance group required some form of physical assistance for mobility or ADL. Dependent patients were those who could not function independently and completely relied on assistance for physical activity and ADL. Length of stay was also tabulated for the rehab and non-rehab group.

Discharge equipment and notes: The following discharge data were obtained: i) discharge equipment (1: none, 2: cane/walker, and 3: hospital bed, Hoyer, wheelchair, or commode (durable medical equipment, DME), or discharge to rehabilitation facility), iii) discharge with or without supplemental oxygen equipment, iv) discharge follow-up recommendations (i.e., cardiology, vascular medicine, pulmonology, endocrinology, neurology, urology, hematology, surgery, gastroenterology, nephrology, psychiatry, ophthalmology, orthopedics/rheumatology, and wound care). Follow-up recommendations of infectious disease and primary care physicians were common to essentially all patients and were not plotted.

Compliance, length of stay_(으), , pre-admission dependency status, and pre-admission domicile with respect to discharge location: Discharge data were obtained for suggested and actual discharge location (1: homecare, 2: rehabilitation facility, 3: long-term care (LTC), or hospice). Compliance, LOS, preadmission domicile status, and pre-admission dependency status with respect to discharge location were analyzed for the rehab and non-rehab group. For pre-admission domicile status, patients were grouped into: i) those from private homes, assisted living facilities (ALF), and group homes, ii) those from a subacute rehab facility (SAR), and iii) those from a skilled nursing facility (SNF).

Functional scores: The modified Mental Status score (range: 0-3) assesses alertness, orientation and ability to follow commands. One point is given if the patient is alert but not oriented, two points are given if the patient is alert and oriented to at least two domains (self, location, time, or situation), and an additional point is given if the patient is able to follow commands. The ICU Mobility Scale (range: 0-10) is an 11-item categorical scale that measures the highest level of functional mobility of patients. Although ICU mobility scale is used in the ICU setting, it is appropriate for this study because COVID-19 patients were constrained to hospital rooms due to COVID-19 circumstances similar to ICU setting. The Barthel Index (original range: $0-100$ ) is an ordinal scale used to measure performance in ADL, consisting of ten variables describing $A D L$ and functional mobility, with a higher number reflecting greater ability to function independently. Due to the isolation precautions for patients with COVID-19, therapy sessions were confined to the patient's room which limited potential ambulation distances. The "mobility on level surfaces" subscale of The Barthel Index (0-15 points) could not be scored as the minimum distance must be 50 yards, greater than what is feasible within the confines of a patient room. Additionally, the "stairs" subscale of the Barthel Index (0-10 points) could not be consistently scored because it was only completed if stairs were a barrier to discharge. Thus, these subscales were eliminated, and the modified Barthel Index used for this study had a range of $0-75$. Higher scores indicate higher functioning for all three scores. 
Functional scores were extracted from patient charts by a team of four occupational therapists and physical therapists (18). Chart review included reviews done by occupational therapy notes, physical therapy notes, nursing flowsheets, care management notes, medicine team notes, and speech-language pathology notes if needed. If specific notes and/or information was not available from the actual date of hospital discharge, the closest note prior to the actual date was used. During these chart reviews, COVID19 diagnosis was confirmed as the final primary diagnosis on the patient chart. Each patient's medical chart was rated by two independent raters (18).

\section{Statistical analysis}

Statistical analysis was performed using SPSS v26 (IBM, Armonk, NY) and SAS v9.4 (SAS Institute, Cary, NC). Chi-square was used to compare categorical demographics, comorbidities, and symptoms at presentation. One sample t-tests were used to compare functional scores to baseline. Age, laboratory tests, vital signs and in-hospital diagnosis were compared using Mann-Whitney U test. Suggested discharge locations were compared with actual discharge locations using McNemar's test. Rehab and non-rehab group differences in pre-admission independency status, LOS and discharge location, discharge equipment, and medical follow-up recommendation used Chi-square analysis.

Functional scores were compared across pre-admission status and the number of days of hospitalization using an ANOVA. Multiple regression models were fit to functional scores with demographics and comorbidities as covariates. Backward selection was utilized, and non-significant comorbidities were removed from the final models. For all analyses, a $p<0.05$ was considered to be statistically significant.

\section{Results}

Of the non-critically ill hospitalized COVID-19 patients, 31.9\% received in-hospital rehab services. Electronic medical record data were extracted for 155 patients who received rehab and 162 patients who did not receive rehab for comparison. Demographics, medical insurance status, comorbidities, symptoms, laboratory tests, and vital signs at hospital admission for the rehab and non-rehab groups are summarized in Table 1. The rehab group were older and had fewer Hispanic's compared to the non-rehab group ( $<<0.05$, Chi square), but there was no sex difference between groups. The majority of non-rehab group (88\%) and rehab group (93\%) had medical insurance. The rehab group had a higher prevalence of previous hypertension, coronary artery disease, immunosuppression, psychiatric disorders, arrythmia's, thromboembolic disorders, and hypothyroidism ( $p<0.05$, Chi-square), but lower prevalence of fever, myalgia, and sore throat ( $<<0.05$, Chi-square) compared to the non-rehab group. The rehab group also had fewer smokers than the non-rehab group. The top five most common comorbidities (obesity, hypertension, smoking, diabetes, and coronary artery disease) were similar in both groups. There were more patients with multiple comorbidities in the rehab group compared to the non-rehab group. The majority of the non-rehab (91\%) and rehab group (92\%) were symptomatic. Of the laboratory tests and vital signs, alanine aminotransferase, brain natriuretic peptide, hematocrit, creatinine, D-dimer, troponin, 
lactate dehydrogenase, lymphocytes, diastolic blood pressure, $\mathrm{SpO}_{2}$, and heart rate were significantly different between groups ( $p<0.05$, Mann-Whitney U Test)

We evaluated the number of patients needing rehabilitation with respect to pre-admission dependency status and found that in the non-rehab group, $82 \%$ were independent, $9 \%$ needed partial assistance and $9 \%$ were dependent, whereas in the rehab group, 59\% were independent, $35 \%$ needed partial assistance, and $6 \%$ were dependent (Figure 1A). Pair-wise comparison showed that more independent patients did not need rehab ( $p<0.001$, Chi-square), more partial assistance patients needed rehab $(p<0.001)$, and a similar number of dependent patients needed rehab $(p>0.05)$.

Length of stay (LOS) was analyzed with respect to dependency status. Overall, the non-rehab group spent fewer days in the hospital compared to the rehab group $(5[3,8]$ vs $9[5,16]$ days, median [IQR], $p<0.0001$, Mann-Whitney U Test). Figure 1B shows days of hospitalization for three bins. There were a higher percentage of patients who were discharged 0-10 days in the non-rehab group than the rehab group (81\% vs $53 \%, p<0.0001$, Chi square), but fewer who were discharged between $10-20$ days $(17 \%$ vs $35 \%, p<0.05)$ and $20+$ days $(2 \%$ vs $12 \%$, $p<0.05)$.

Discharge equipment was evaluated for the rehab and non-rehab group. Compared to the non-rehab patients, fewer rehab patients were discharged with no-equipment ( $38 \%$ vs $83 \% \mathrm{p}<0.001$ ), whereas more rehab patients were discharged with a cane or rolling walker ( $18 \%$ vs $1 \%, p<0.001)$, and with DME ( $44 \%$ vs $15 \%, p<0.001$ for all, Chi-square, Figure 2A). More rehab patients were discharged with oxygen equipment than the non-rehab group ( $35 \%$ vs $16 \%$ respectively, $p<0.01$, Chi-square, Figure 2 B).

We evaluated follow-up referrals in the discharged patients. Overall, comparatively fewer referrals were observed in the non-rehab group. Cardiology, vascular medicine, urology, and gastroenterology follow-up referrals were among the top six recommendations for rehab group (Figure 3A). Cardiology, vascular medicine, endocrinology, pulmonology, and hematology referrals were significantly different between groups ( $p<0.05$, Chi-square). Rehab patients were more likely to have multiple referrals while the nonrehab group was more likely to have no referrals ( $p<0.001$, Chi square, Figure $3 \mathbf{B}$ ).

Compliance to discharge location and length of stay with respect to discharge locations were analyzed. Most patients adhered to their suggested discharge recommendation ( $p>0.05$, McNemar's test, Figure 4A). Of those who did not, $38 \%$ elected a higher standard of care than suggested and $62 \%$ elected a lower standard of care. About half (52\%) of the patients were discharged to home with home care, $42 \%$ were discharged to rehab facility and $<10 \%$ were discharged to LTC/hospice.

Patients discharged to homecare spent significantly less time in the hosptial compared to those dicharged to rehab (9.4 vs 13.8 days, $p<0.05$, ANOVA, Figure 4B). There was no significant difference in LOS between discharge location to rehab and LTC/hospice $(p>0.05)$ or between between discharge location to homecare and LTC/hospice $(p>0.05)$. 
Pre-admission domicile was analyzed with respect to discharge locations for the rehab group. (Figure 4C). For patients recommended discharge to homecare or rehab, the majority ( $96 \%$ and $90 \%$ respectively) came from private homes, assisted living facilities (ALF), and group homes, while a few came from subacute rehab facilities (SAR; $2 \%$ and $6 \%$ ) or skilled nursing facilities (SNF; $1 \%$ and $3 \%$ ). By contrast, for patients recommended discharge to LTC/Hospice, most (80\%) came from a SNF. By comparison essentially all patients in the non-rehab group returned to their prior domicile.

Pre-COVID-19 independent status was analyzed with respect to discharge locations for the rehab group (Figure 4D). For survivors recommended homecare, the majority were functionally independent pre COVID-19 (78.8\%), some needed partial assistance (18.8\%), and very few (2.5\%) were dependent. For survivors recommended rehab, similar number of patients were functionally independent (43\%) and needing partial assistance (54\%), and very few (3\%) were dependent. For survivors recommended long term care or hospice, none were functionally independent, and half needed partial assistance $(50 \%)$ or were dependent (50\%).

\section{Functional scores}

Functional scores were evaluated with respect to pre-admission dependency status and LOS. Functional scores of rehab patients were below normal (Mental Status Score: 2.72 out of 3, ICU Mobility Scale: 6.87 out of 10, and modified Barthel Index Score: 45.58 out of 75 , all $p<0.0001$ one-sample t-test). Functional scores relied on pre-admission status, with higher scores in patients who were independent preadmission, followed by those who required partial assistance and were dependent $(p<0.0001$ for all scores, ANOVA, Figure 5A). Functional scores did not depend on duration of hospitalization $(p>0.05$, ANOVA, Figure 5B).

Table 2 shows the correlation of Mental Status, ICU Mobility Scale, and modified Barthel Index scores at hospital discharge with demographics, comorbidities, laboratory tests, and vitals. Most of the Barthel scores were significantly negatively correlated with age and hypertension, coronary artery disease, chronic kidney disease, psychiatric disease, and anemia, and neurological disorders $(p<0.05$, ANOVA), whereas only some mobility and mental status scores were significantly correlated $(p<0.05$, ANOVA). Functional scores were not significantly correlated with lung disorders, carcinoma, hyperlipidemia, gender, race, or ethnicity.

\section{Discussion}

[pre-admission, age, comorbidity] About a third of non-critically ill COVID-19 patients in our cohort received in-hospital rehabilitative services. The actual number needing in-hospital rehabilitation is likely higher because some patients were recommended but did not receive rehab service given the COVID-19 circumstances and concerns for cross infection.

In-hospital rehabilitation was found to be associated with pre-COVID-19 dependency status, with partial assistance or dependent patients showing higher prevalence of rehabilitation than independent patients. 
It is not surprising that older age and some comorbidities were associated with higher prevalence of inhospital rehabilitation. The contribution of pre-COVID-19 dependency status, age, and comorbidity are likely related and may not be independent factors accounting for rehab needs. Pre-COVID-19 dependency status, older age and comorbidities contributed to worse disease severity (as indicated by vital signs and laboratory tests) and longer hospital stay in the rehab group. Note that duration of hospitalization could be affected by rehab facility's policy requiring patients to be COVID-19 negative for admission and, thus, some COVID-19 patients might have remained in the hospital longer than medically necessary.

Discharge equipment and medical recommendations: Rehab patients were discharged with more assistive equipment and oxygen equipment compared to non-rehab patients. The rehab group had markedly more follow-up referrals at discharge than the non-rehab group which is not unexpected because rehab patients had worse disease severity. Notably, the rehab group had a higher percentage of cardiology, vascular medicine, endocrinology, and pulmonology follow-up recommendations at discharge. Most of these referrals appeared to be a result of COVID-19 or worsened by COVID-19 illness, although some might be related to pre-admission comorbidities. For example, vascular medicine referral might be a concern over hypercoagulability of COVID-19 (19) and cardiology referral might be related to concerns of SARS-CoV2 virus damage to the heart which has high density of ACE2 receptors through which SARSCoV2 infects cells (20). The rehab group were discharged with more durable medical equipment, consistent with worse functional outcomes. About $35 \%$ of rehab group and $15 \%$ of the non-rehab group were discharged with oxygen, consistent with findings that respiratory function was still compromised by COVID-19 at discharge.

Domicile and discharge locations: Many non-critically ill COVID-19 survivors, including those who were independent prior to COVID-19 illness, were recommended rehabilitation or upgraded care at discharge. Patients who were functionally independent pre COVID-19 were more likely to be discharged to homecare, but significant number of these patients were also sent to rehabilitation facility. In the rehab group, there were more patients recommended rehabilitation or upgraded care at discharge. In stark contrast, essentially all of patients in the non-rehab group returned to their prior domicile. The actual discharge locations could be affected by infection concerns or rehab facility availability.

Functional Scores: In-hospital functional assessment was constrained by COVID-19 circumstances. Patients were usually confined to their hospital rooms to reduce cross-infection, and thus ambulation distance for functional assessment was limited. Many hospitals, including ours, needed to increase bed capacity during the peak of COVID-19, resulting in shortage of some rehab equipment and reduction in room space, making rehabilitation challenging. The COVID-19 circumstance also confined patients to beds, limiting mobility and resulting in worse functional scores at discharge which likely negatively impact long-term physical recovery. Due to these constraints, certain components of Barthel Index could not be scored and thus the modified Barthel Index used in this study only had a range of 0-75

The average Mental Status (2.65 out of 3), ICU Mobility Scale (6.59 out of 10), Barthel Index (40.07 out $75)$ scores at discharge were below normal at discharge. There was an association of functional scores 
with pre-admission dependency status, in which partial assistance or dependent patients had lower functional scores. These observations indicated functional scores were related to pre-existing conditions or worsened by COVID-19 illness due to pre-existing conditions. Functional scores were not correlated with LOS. A possible explanation is that patients likely needed to be able to perform basic tasks before they were discharged, especially given that rehabilitation facilities and home likely required that patients were COVID-19 free.

Some Mental Status, some ICU Mobility Scale and most Barthel Index scores were negatively correlated with age and hypertension, coronary artery disease, chronic kidney disease, psychiatric disease, and anemia, and neurological disorders. No other correlations were found between functional scores with other clinical variables. These findings suggested that functional scores were associated with pre-existing conditions and made worse by COVID-19. Follow-up studies are needed to ascertain the contribution of pre-existing conditions.

Comparison with critically ill COVID-19 patients at discharge: We previously reported the functional status of critically ill COVID-19 patients at discharge (18). In that study, the majority of (94.1\%) were functionally independent prior to COVID-19 illness, whereas the majority of critically ill COVID-19 patients were not functionally independent at hospital discharge (22\% discharged with cane or rolling walker, $49 \%$ discharged with durable medical equipment. By comparison, $80 \%$ of the non-rehab and $58 \%$ of rehab group were functionally independent prior to COVID-19 illness. It is unclear why there were differences in pre-COVID-19 independency status between the critically ill and noncritically ill COVID-19 groups. It is possible that those who did not survive in the critically ill group were mostly dependent or needing partial assistance, whereas those who were functionally independent prior to COVID-19 illness were those who survived.

There were more critically ill COVID-19 patients (50\%) discharged with supplemental oxygen equipment compared to noncritically ill COVID-19 patients (35\%). Markedly more critically ill COVID-19 patients (62.71\%) were discharged with hospital bed, Hoyer, wheelchair, or commode (durable medical equipment, DME), or discharged to rehabilitation facility, compared to both noncritically ill COVID-19 patients rehab and non-rehab group. Similar percentage of critically ill COVID-19 patients were discharged with cane or rolling walker compared to the noncritically ill rehab group. Few critically ill COVID-19 patients (15.25\%) were discharged with no assisted equipment, compared to the majority of the noncritically ill COVID-19 patients were discharged with no assisted equipment.

Comparatively more critically ill COVID-19 patients were referred for medical follow-up recommendation but the types of top medical follow-up recommendations were similar to noncritically ill COVID-19 patients, consistent with COVID-19 related illness or concerns associated with COVID-19 disease.

The functional status of critically ill COVID-19 patients (Mental status $=2.7$, ICU Mobility Scale $=5.75$, and Barthel index $=38$ ) showed slightly worse scores using the same tests compared to that of noncritically ill COVID-19 patients (ICU mental status $=2.72$, ICU mobility scale $=6.87$, and Barthel index $=$ 48.58), as expected. Worse functional status at hospital discharge in critically ill COVID-19 was 
associated with longer invasive mechanical ventilation duration, older age, male sex, higher number of comorbidities, hypertension, diabetes, chronic obstructive pulmonary disease, and immunosuppression. Taken together these comparisons indicate that critically ill COVID-19 patients were in worse shape at discharge, were discharged with more DME equipment, and required markedly more medical follow-up.

\section{Conclusions}

This study investigated the extent of in-hospital rehabilitation and degree of functional status of COVID19 non-critically ill survivors with respect to pre-admission dependency status, clinical and laboratory test data, length of hospitalization, medical follow-up recommendation, discharged durable medical equipment (DME), discharge location, amongst others. Significant number of non-critically ill COVID-19 survivors received in-hospital rehabilitation. Many non-critically ill COVID-19 survivors showed impaired functional status at hospital discharge and were recommended for upgraded care and medical follow-up

after discharge. Functional scores were associated with pre-admission dependency with some functional scores were negatively correlated with age and hypertension, coronary artery disease, chronic kidney disease, psychiatric disease, and anemia, and neurological disorders. These findings need to be replicated in a larger sample size and across multiple hospitals to achieve generalizability. Prospective studies are also warranted to follow up COVID-19 patients after hospital discharge as many patients will likely have significant post-acute COVID-19 sequela.

\section{Declarations}

\section{Acknowledgements}

We thank all the doctors, nurses and clinical scientists who worked in the hospital during the period of patient recruitment as well as the patients who were involved in this study.

\section{Author contributions}

BM, LB, RJ, MM, HB, WH, conceptualized, collected and analyzed data, drafted paper

TQD conceptualized, wrote paper, edited paper, and supervised.

\section{Competing interests}

All authors declare that they have no competing interests.

\section{References}

1. Zhu, N. et al. China Novel Coronavirus I, Research T. A Novel Coronavirus from Patients with Pneumonia in China, 2019. N Engl J Med. 382 (8), 727-733 (2020).

2. Huang, C. et al. Clinical features of patients infected with 2019 novel coronavirus in Wuhan. China. Lancet. 395 (10223), 497-506 (2020). 
3. Schweickert, W. D. et al. Early physical and occupational therapy in mechanically ventilated, critically ill patients: a randomised controlled trial. Lancet. 373 (9678), 1874-1882 (2009).

4. Liu, K. et al. Respiratory rehabilitation in elderly patients with COVID-19: A randomized controlled study. Complement Ther Clin Pract. 39, 101166 (2020).

5. Li, J. Rehabilitation management of patients with COVID-19: lessons learned from the first experience in China. Eur J Phys Rehabil Med. 56 (3), 335-338 (2020).

6. Sessler, C. N. et al. The Richmond Agitation-Sedation Scale: validity and reliability in adult intensive care unit patients. Am J Respir Crit Care Med. 166 (10), 1338-1344 (2002).

7. Hodgson, C. et al. Feasibility and inter-rater reliability of the ICU Mobility Scale. Heart Lung. 43 (1), 19-24 (2014).

8. Duffy, L., Gajree, S., Langhorne, P., Stott, D. J. \& Quinn, T. J. Reliability (inter-rater agreement) of the Barthel Index for assessment of stroke survivors: systematic review and meta-analysis. Stroke. 44 (2), 462-468 (2013).

9. Sheehy, L. M. Considerations for Postacute Rehabilitation for Survivors of COVID-19. JMIR Public Health Surveill. 6 (2), e19462 (2020).

10. Wang, T. J. et al. Physical Medicine and Rehabilitation and Pulmonary Rehabilitation for COVID-19. Am J Phys Med Rehabil. 99 (9), 769-774 (2020).

11. Stam, H. J., Stucki, G. \& Bickenbach, J. European Academy of Rehabilitation M. Covid-19 and Post Intensive Care Syndrome: A Call for Action. J Rehabil Med. 52 (4), jrm00044 (2020).

12. Rooney, S., Webster, A. \& Paul, L. Systematic Review of Changes and Recovery in Physical Function and Fitness After Severe Acute Respiratory Syndrome-Related Coronavirus Infection: Implications for COVID-19 Rehabilitation. Phys Ther. 100 (10), 1717-1729 (2020).

13. Li, Z. et al. Rehabilitation needs of the first cohort of post-acute COVID-19 patients in Hubei, China. Eur J Phys Rehabil Med. 56 (3), 339-344 (2020).

14. Kiekens, C. et al. Rehabilitation and respiratory management in the acute and early post-acute phase. "Instant paper from the field" on rehabilitation answers to the COVID-19 emergency. Eur J Phys Rehabil Med. 56 (3), 323-326 (2020).

15. Demeco, A. et al. Rehabilitation of patients post-COVID-19 infection: a literature review. $J$ Int Med Res. 48 (8), 300060520948382 (2020).

16. Carda, S. et al. COVID-19 pandemic. What should Physical and Rehabilitation Medicine specialists do? A clinician's perspective. Eur J Phys Rehabil Med. 56 (4), 515-524 (2020).

17. Barker-Davies, R. M. et al. The Stanford Hall consensus statement for post-COVID-19 rehabilitation. Br J Sports Med. 54 (16), 949-959 (2020).

18. Musheyev, B. et al. Functional status of mechanically ventilated COVID-19 survivors at ICU and hospital discharge. J Intensive Care. 9 (1), 31 (2021).

19. Bajgain, K. T., Badal, S., Bajgain, B. B. \& Santana, M. J. Prevalence of comorbidities among individuals with COVID-19: A rapid review of current literature. Am J Infect Control. 49 (2), 238-246 
(2021).

20. Hoffmann, M. et al. SARS-CoV-2 Cell Entry Depends on ACE2 and TMPRSS2 and Is Blocked by a Clinically Proven Protease Inhibitor. Cell. 181 (2), 271-280 e278. (2020).

\section{Tables}

Table 1. Demographics, comorbidities, symptoms, laboratory tests, and vitals in the rehab $(\mathrm{N}=155)$ and non-rehab ( $\mathrm{N}=162)$ group at hospital admission. Group comparison of categorical variables in percentages used Chi-square tests. Group comparison of continuous variables in medians and interquartile ranges (IQR) used the Mann-Whitney $U$ test. Bolded values indicate significance. Functional scores are represented as mean [SEM]. $P$ values in bold indicate statistical significance. N.A. indicates not available. COPD: chronic obstructive pulmonary disease, GI disease: Gastrointestinal disease. 


\section{Demographics}

\begin{tabular}{|c|c|c|c|}
\hline Age & $76.00(63.00,83.00)$ & $51.50(38.50,60.00)$ & 0.00 \\
\hline \multicolumn{4}{|l|}{ Gender } \\
\hline Male & $48.39 \%$ & $57.41 \%$ & 0.11 \\
\hline Female & $51.61 \%$ & $42.59 \%$ & \\
\hline \multicolumn{4}{|l|}{ Ethnicity } \\
\hline Hispanic & $16.77 \%$ & $30.25 \%$ & 0.01 \\
\hline Non-Hispanic & $71.61 \%$ & $56.17 \%$ & 0.00 \\
\hline \multicolumn{4}{|l|}{ Race } \\
\hline Caucasian & $50.32 \%$ & $51.23 \%$ & 0.87 \\
\hline African American & $3.87 \%$ & $7.41 \%$ & 0.17 \\
\hline Insurance & $92.90 \%$ & $88.27 \%$ & 0.16 \\
\hline \multicolumn{4}{|l|}{ Comorbidities } \\
\hline Hypertension & $61.29 \%$ & $40.74 \%$ & 0.00 \\
\hline Smoking & $33.55 \%$ & $47.53 \%$ & 0.02 \\
\hline Coronary Artery Disease & $19.35 \%$ & $10.49 \%$ & 0.03 \\
\hline Immunosuppression & $13.55 \%$ & $4.32 \%$ & 0.00 \\
\hline Psychiatric Disorder & $12.26 \%$ & $5.56 \%$ & 0.04 \\
\hline Arrythmia & $10.97 \%$ & $3.70 \%$ & 0.01 \\
\hline Hypothyroidism & $7.10 \%$ & $0.62 \%$ & 0.00 \\
\hline Thromboembolic Disorders & $7.10 \%$ & $1.23 \%$ & 0.01 \\
\hline Obesity & $74.83 \%$ & $83.69 \%$ & 0.06 \\
\hline Diabetes & $29.03 \%$ & $20.99 \%$ & 0.10 \\
\hline Carcinoma & $14.84 \%$ & $9.88 \%$ & 0.18 \\
\hline COPD & $11.61 \%$ & $9.26 \%$ & 0.49 \\
\hline Heart Failure & $11.61 \%$ & $6.17 \%$ & 0.09 \\
\hline Chronic Kidney Disease & $10.97 \%$ & $11.11 \%$ & 0.97 \\
\hline Hyperlipidemia & $7.74 \%$ & $3.70 \%$ & 0.12 \\
\hline
\end{tabular}




\begin{tabular}{|c|c|c|c|}
\hline Asthma & $6.45 \%$ & $3.70 \%$ & 0.26 \\
\hline GI Disease & $5.81 \%$ & $7.41 \%$ & 0.57 \\
\hline \multicolumn{4}{|l|}{ Symptoms } \\
\hline Fever & $53.55 \%$ & $67.28 \%$ & 0.01 \\
\hline Myalgia & $14.84 \%$ & $30.25 \%$ & 0.00 \\
\hline Sore Throat & $1.29 \%$ & $5.56 \%$ & 0.04 \\
\hline Shortness of Breath & $54.84 \%$ & $60.49 \%$ & 0.31 \\
\hline Cough & $50.97 \%$ & $60.49 \%$ & 0.09 \\
\hline Fatigue & $26.45 \%$ & $22.84 \%$ & 0.46 \\
\hline Nausea/Vomiting & $23.23 \%$ & $24.07 \%$ & 0.86 \\
\hline Diarrhea & $18.71 \%$ & $20.37 \%$ & 0.71 \\
\hline Chest Discomfort/Pain & $11.61 \%$ & $16.05 \%$ & 0.25 \\
\hline Sputum & $5.81 \%$ & $6.79 \%$ & 0.72 \\
\hline Headache & $4.52 \%$ & $8.64 \%$ & 0.14 \\
\hline Runny Nose & $3.87 \%$ & $2.47 \%$ & 0.48 \\
\hline Loss of Taste & $3.87 \%$ & $3.70 \%$ & 0.94 \\
\hline Loss of Smell & $2.58 \%$ & $3.09 \%$ & 0.79 \\
\hline Asymptomatic & $8.39 \%$ & $9.26 \%$ & 0.78 \\
\hline \multicolumn{4}{|l|}{ Laboratory Tests } \\
\hline Alanine Aminotransferase, $\mathrm{U}, \mathrm{L}$ & $21.5(12.0,37.5)$ & $34(20.5,66.5)$ & 0.01 \\
\hline Brain Natriuretic Peptide, ng/L & $529(148,1346)$ & $163(38.0,540.5)$ & 0.00 \\
\hline Hematocrit, \% & $38.5(33.4,41.9)$ & $40.2(37.0,44.7)$ & 0.00 \\
\hline Creatinine, mg/dL & $1.29(0.84,35.57)$ & $1.06(0.72,5.73)$ & 0.00 \\
\hline D-Dimer, nmol/L & $412(240,1027)$ & $317(201.75,946)$ & 0.00 \\
\hline Troponin, $\mu \mathrm{g} / \mathrm{L}$ & $0.01(0.01,0.03)$ & $0.01(0.01,0.01)$ & 0.01 \\
\hline Lactate Dehydrogenase, U/L & $333(269,401)$ & $351(263,434)$ & 0.02 \\
\hline Lymphocytes, \% & $12.3(9.5,16.5)$ & $13(7.25,18.6)$ & 0.02 \\
\hline Procalcitonin, $\mathrm{ng} / \mathrm{mL}$ & $0.16(0.10,0.24)$ & $0.22(0.1075,0.325)$ & 0.08 \\
\hline Bicarbonate, $\mathrm{mEq} / \mathrm{L}$ & $24(22,26)$ & $23(21.25,25.5)$ & 0.26 \\
\hline
\end{tabular}




\begin{tabular}{|c|c|c|c|}
\hline Sodium, $\mathrm{mEq} / \mathrm{L}$ & $137.5(133,140)$ & $136(130.75,139.25)$ & 0.31 \\
\hline Aspartate Aminotransferase, U/L & $38(24,55)$ & $41(26.5,57.75)$ & 0.38 \\
\hline White Blood Cells, G/L & $6.63(5.01,8.96)$ & $7.77(5.59,9.72)$ & 0.49 \\
\hline C-reactive Protein, $\mathrm{mg} / \mathrm{L}$ & $6.5(3.4,12.3)$ & $6.7(2.45,15.4)$ & 0.51 \\
\hline Ferritin, $\mu \mathrm{g} / \mathrm{L}$ & $635.4(228.7,1234)$ & $796(384.15,1163)$ & 0.74 \\
\hline \multicolumn{4}{|l|}{ Vitals } \\
\hline Diastolic Blood Pressure, $\mathrm{mmHg}$ & $72.67(64.57,78.56)$ & $72.67(68.25,77.43)$ & 0.02 \\
\hline $\mathrm{SpO}_{2}, \%$ & $95.27(94,96.56)$ & $95.91(94.65,97.13)$ & 0.02 \\
\hline Heart Rate, bpm & $82.67(71.5,94.8)$ & $92.33(73.375,106.9)$ & 0.01 \\
\hline Systolic Blood Pressure, mmHg & $130(123.5,144.571)$ & $122.5(115.341,129.167)$ & 0.08 \\
\hline Respiratory Rate, rate/min & $19.3(17.5556,21.0909)$ & $20(18.0834,24.2798)$ & 0.16 \\
\hline Temperature, ${ }^{\circ} \mathrm{C}$ & $37.05(36.8,37.4667)$ & $37.34(36.9,37.8)$ & 0.07 \\
\hline In-hospital diagnosis & $10.32 \%$ & $9.26 \%$ & 0.75 \\
\hline \multicolumn{4}{|l|}{ Acute Kidney Injury } \\
\hline Acute Respiratory Failure & $20.00 \%$ & $12.96 \%$ & 0.09 \\
\hline \multicolumn{4}{|c|}{ Functional scores [standard error of the mean] } \\
\hline Mental Status & $2.72[0.05]$ out of 3 & N.A. & N.A. \\
\hline ICU Mobility & $6.87[0.21]$ out of 10 & N.A. & N.A. \\
\hline Barthel Index & 45.58 [2.05] out of 75 & N.A. & N.A. \\
\hline
\end{tabular}

Table 2. Regression coefficients (standard errors) and $P$ values of functional status scores with demographics, comorbidities, laboratory values, and vital signs. Correlations for comorbidities that had $<6 \%$ prevalence were not analyzed as they were unreliable. ns: no significance. 


\begin{tabular}{|llll|}
\hline & Mental Status Score & Mobility Scale & Barthel Index \\
\hline Hypertension & $0.225(2.882) 0.005$ & ns & ns \\
\hline Coronary Artery Disease & ns & ns & $0.144(2.134) 0.035$ \\
\hline Chronic Kidney Disease & $-0.195(-2.607) 0.01$ & $n s$ & $-0.158(-2.345) 0.021$ \\
\hline Psychiatric Disorder & $-0.326(-4.329) 0$ & $n s$ & $-0.15(-2.276) 0.024$ \\
\hline Anemia & ns & $-0.176(-2.287) 0.024$ & $-0.181(-2.625) 0.01$ \\
\hline Neurological Disorders & ns & $-0.239(-3.221) 0.002$ & $-0.181(-2.784) 0.006$ \\
\hline Age & $-0.269(-3.157) 0.002$ & $-0.45(-5.477) 0$ & $-0.503(-6.988) 0$ \\
\hline
\end{tabular}

\section{Figures}

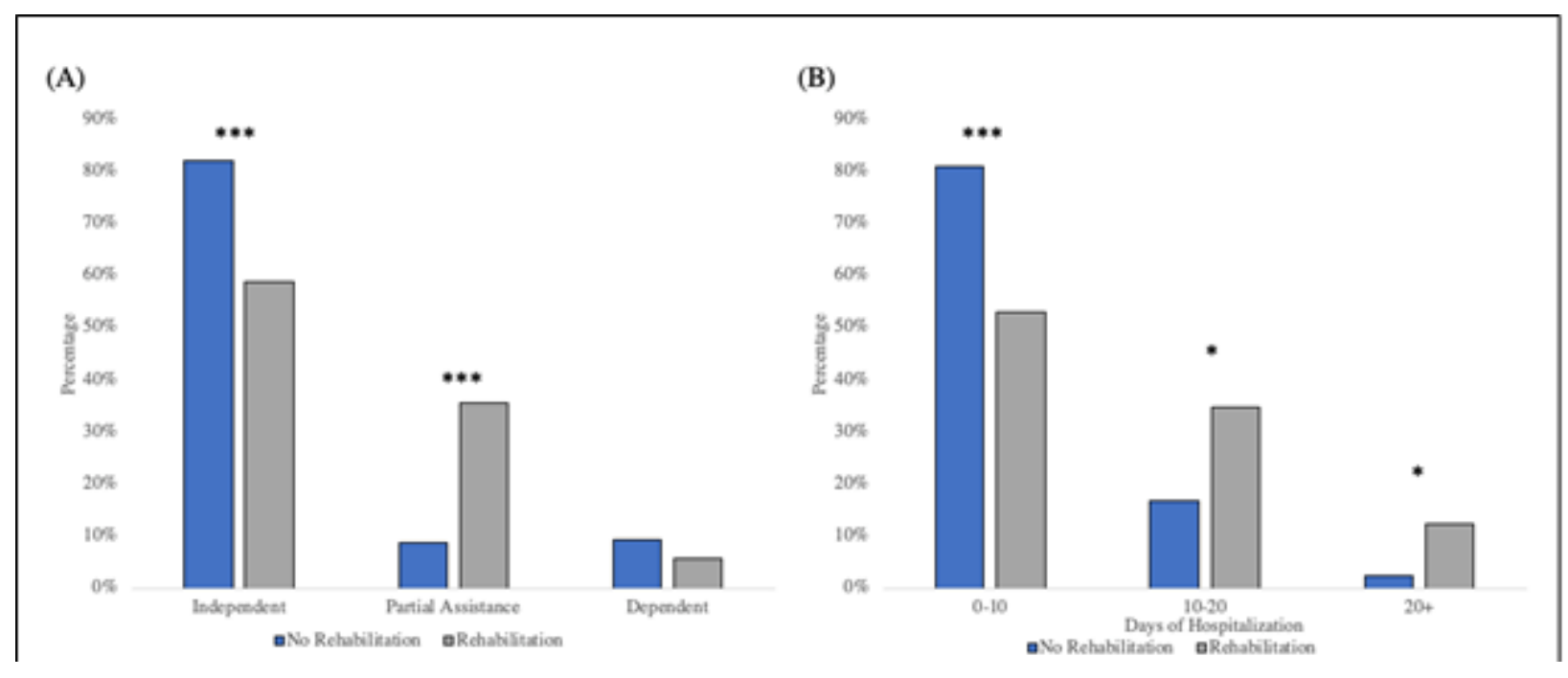

Figure 1

(A) Percentage of patients that were independent, dependent, or required partial assistance in the nonrehab and rehab group. (B) Percentage of patients in the non-rehab and rehab group that were hospitalized for less than 10 days, 10-20 days, or more than 20 days. *** indicates significance $p<0.001$, * indicates $p<0.05$ (Chi square). 


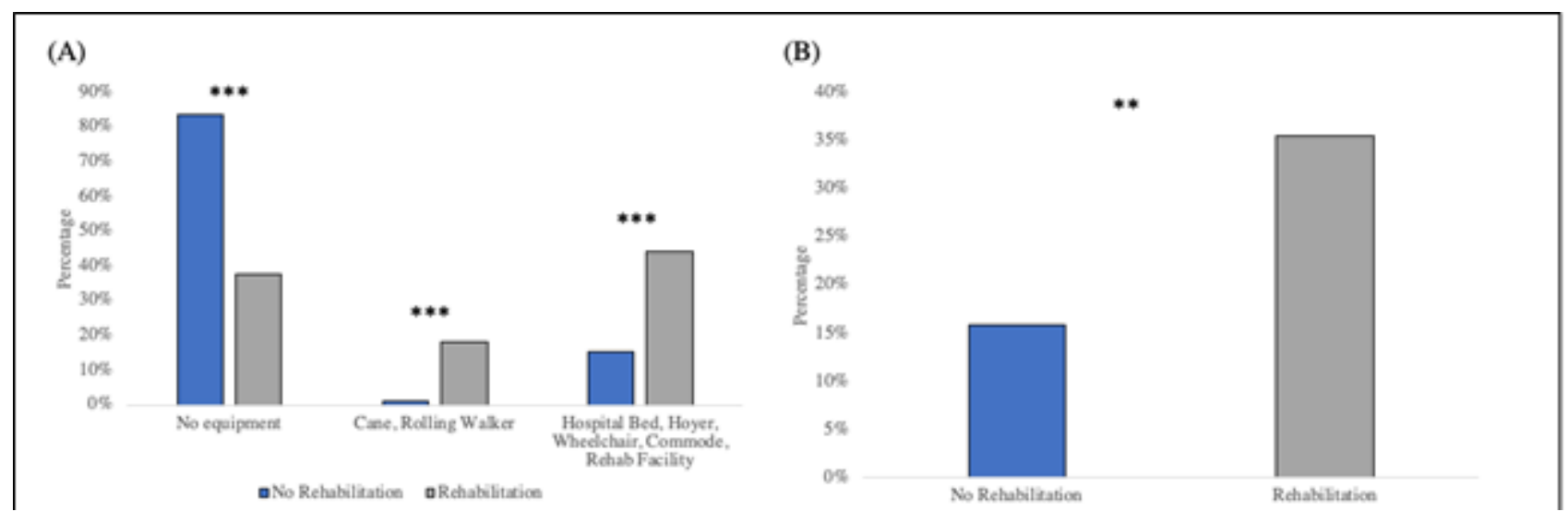

Figure 2

(A) Percentage of patients in the non-rehab and rehab group discharged: i) with no equipment, ii) with cane or rolling walker, iii) with hospital bed, Hoyer, wheelchair, and/or commode (also referred to as durable medical equipment, DME), and to rehabilitation facility. (B) Patients discharged with or without supplemental oxygen equipment. *** indicates $p<0.001$, ** indicates $p<0.01$ (Chi Square).

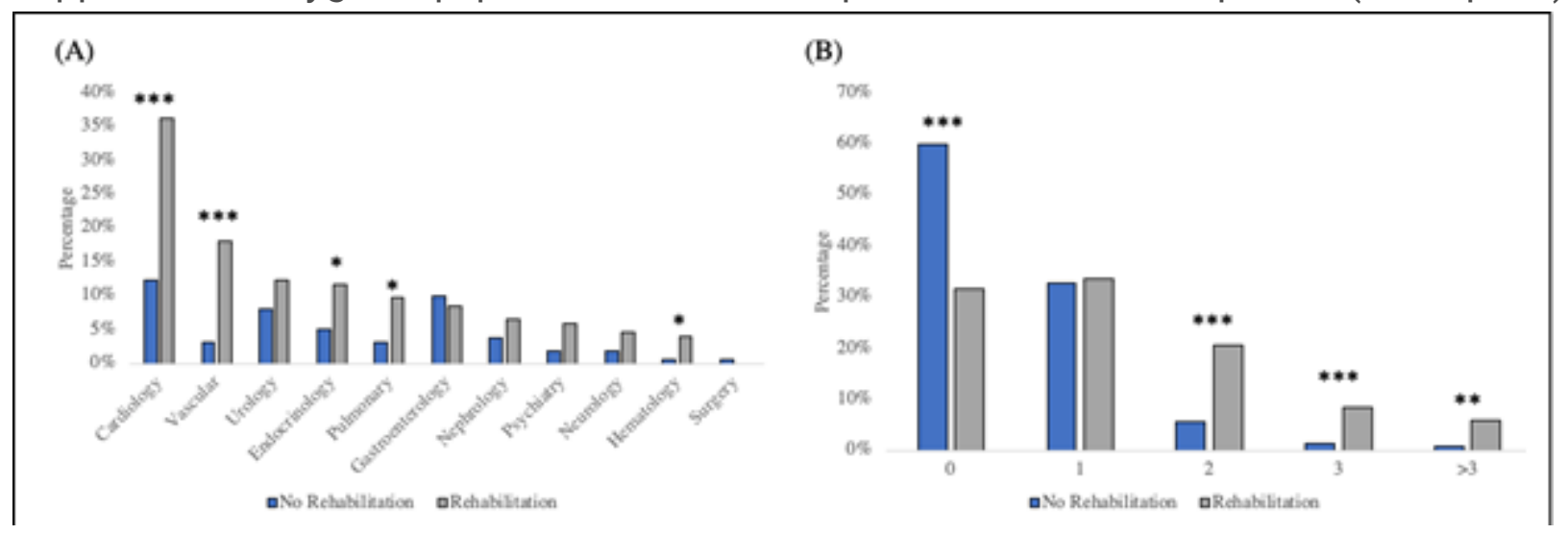

\section{Figure 3}

(A) Histogram of medical follow-up recommendations, and (B) percentages of patients with number of follow-up recommendations. *** indicates $p<0.001$, ** indicates $p<0.01$, * indicates $p<0.05$ (Chi square)

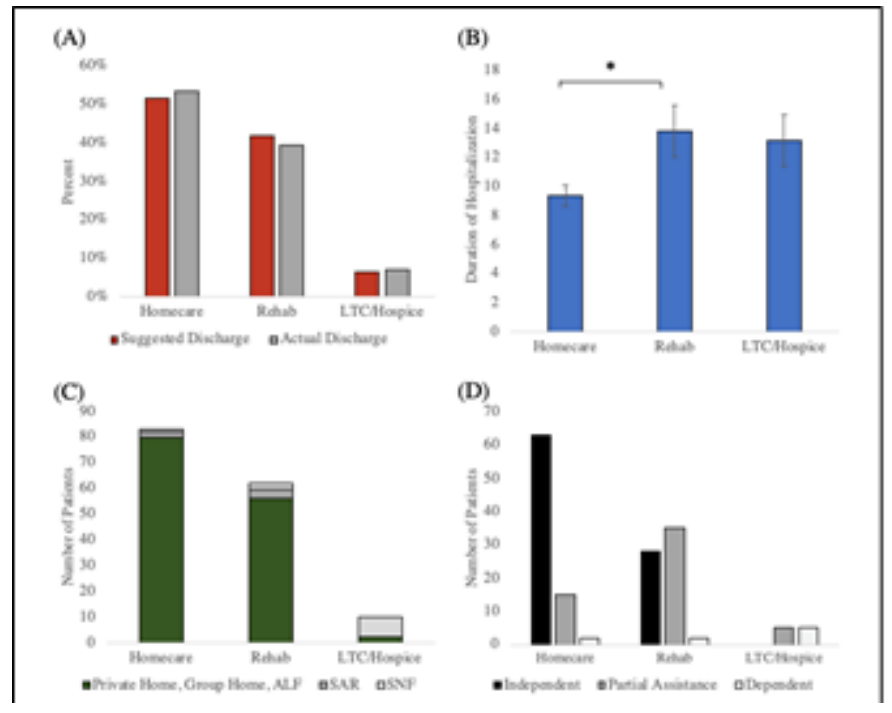


Figure 4

(A) Discharge compliance, (B) duration of hospitalization versus suggested discharge location, (C) preadmission domicile versus actual discharge location, and (D) pre-admission independent status versus suggested discharge location. * indicates $p<0.05$ (Chi Square). ALF: assisted living facility, SAR: subacute assisted rehab, SNF: skilled nursing facility.

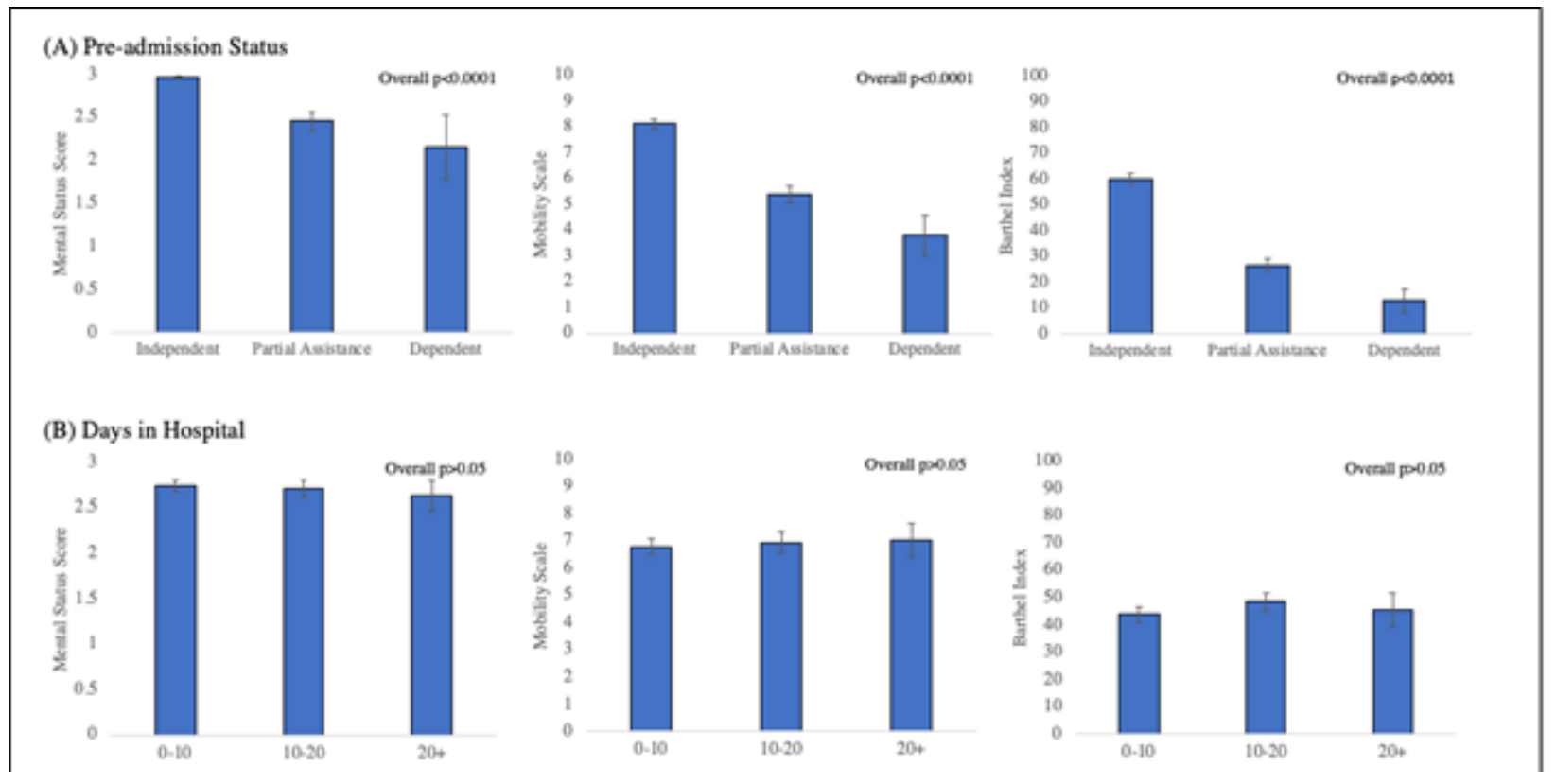

Figure 5

Modified Mental Status Score (range: 0-3), ICU Mobility Scale (range: 0-10), and modified Barthel Index (range: $0-75$ ) for (A) pre-admission dependency status (overall $p<0.001$, ANOVA) and (B) duration of hospitalization (overall $p>0.05$, ANOVA). 